\title{
A REALIDADE DA PESQUISA NO DEN/UFS 1
}

\author{
THE TRUTH ABOUT THE RESEARCH ON DEN/UFS \\ LA REALIDAD DE LA PESQUISA EN EL DEN/UFS
}

\section{Delvair de Brito Alves² Lindete Amorim Santos ${ }^{3}$}

RESUMO: Esta pesquisa trata do processo de pesquisar no Departamento de Enfermagem e Nutrição da Universidade Federal de Sergipe - DEN/UFS. Ela foi desenvolvida a partir de entrevistas semi estruturadas realizadas com as docentes. Dos materiais obtidos, apresentamos concepções das docentes sobre grupos, linhas e projetos de pesquisa; a produção científica docente e discente; dificuldades e possibilidades relativas à atividade de pesquisar; estratégias que esse Departamento vem utilizando para o desenvolvimento da pesquisa, para a capacitação docente e formação de pesquisadores, além da inserção de estudantes (de graduação e de pós graduação) no "mundo científico". Ela conclui pela necessidade de aprofundamento dessa atividade e pela divulgação e aplicação dos seus resultados na prática de enfermagem.

PALAVRAS - CHAVE: Pesquisa, Grupo de pesquisa, Linha de pesquisa, Projeto de pesquisa

\begin{abstract}
This study is about the research process in the Nursing and Nutrition Department of the Federal University of Sergipe - DEN/UFS. It was developed through semi structured interviews made with the professors. From the material obtained, we presented the professor's conceptions about groups, areas and research projects; the scientific production; difficulties and possibilities related to the research activity; strategies that this Department has been using for the development of research, the capacitation of professors and the researchers' formation, besides the initiation of students (graduation and pos graduation) in the "scientific world". As its result, we got the need of deepening in this activity and the propagation and application of its results in nursing.
\end{abstract}

KEY WORDS: Research, Research group, Research area, Research project

RESUMEN: Esta pesquisa trata al respecto del proceso pesquisa en el Departamento de Enfermeria y Nutrición de la Universidad Federal de Sergipe-DEN/UFS. Fue desarrollada partir de entrevistas semiestructuradas realizadas com los profesores. De los materiales obtenidos, presentamos la concepción de los profesores sobre grupos, líneas y proyectos de pesquisa, producción científica, dificultades y posibilidades relativas a la actividad de pesquisa, la capacitación de profesores y la formación de pesquisadores, además de la incorporación de estudiantes( graduación y postgraduación) en el "mundo científico". Como conclusión, sentimos la necesidad de profundizarnos en esta actividad, divulgar y aplicar sus resultados en la prácti a de enfermería.

PALABRAS CLAVE: Pesquisa, grupo de pesquisa, línea de pesquisa, proyectos de pesquisa

\footnotetext{
TTrabalho apresentado na "Semana Comemorativa dos 20 Anos do Curso de Enfermagem da Universidade Federal de Sergipe", que aconteceu durante as comemorações da Semana Brasileira de Enfermagem.

${ }^{2}$ Enfermeira, Mestre e Dra em Educação, Professor Visitante do Núcleo de Pós-Graduação em Enfermagemda Universidade Federal de Sergipe.

${ }^{3}$ Enfermeira, Mestre e Dra. em Enfermagem, Chefe do Departamento de Enfermagem e Nutrição da UFS e Coordenadora do Núcleo de Pós-Graduação em Enfermagem desta Universidade.
} 


\section{INTRODUÇÃO}

Este estudo trata da pesquisa enquanto uma das atividades do Departamento de Enfermagem e Nutrição da Universidade Federal de Sergipe - DEN/UFS. Ele foi desenvolvido no período de novembro de 1996 a abril de 1997, através de entrevistas realizadas com docentes do quadro permanente desse Departamento.

Esse Departamento, criado em 1976, vive, portanto, no período em que embora a pesquisa seja tratada como fato social, como processo coletivo, a enfermagem encontra-se (como muitas outras áreas) no estágio de aprender a pesquisar.

Mas, o que significa pesquisar? Pesquisa indica um momento parcial do processo mais geral de conhecer, descrever e explicar a realidade em seus fragmentos ou no seu todo. No sentido mais geral, ...'pesquisa' equiivale a 'investigação científica', razão pela qual 'pesquisador' e 'cientista' são termos intercambiáveis, ao menos na linguagem corrente... como sugere Gonçalves (1985, p. 34).

Na enfermagem brasileira, o início da pesquisa aconteceu por volta de 1970, com a criação do regime de tempo integral e dedicação exclusiva para o corpo docente, sendo esta atividade incrementada a partir de 1972 com a criação dos cursos de pós graduação em enfermagem. De acordo com Souza (1988, p. 96), isto mostra ...que a investigação em enfermagem, além de incipiente, é marcada por um processo restrito de divulgação de resultados, o que desvitaliza o seu potencial de desenvolvimento e, em consequiência, o processo de consolidação do conhecimento em enfernagem.

Para Clark; Hockey (1989) As investigações em enfermagem durante os últimos 22 anos [entre 1967 e 1989] contribuíram para a teoria de un corpo de enfermagem em crescinlento, mas falharam, de uma maneira geral, em influenciar a prática clínica. Estes autores concluem que os cuidados e a prática na enfermagem são "comandados" pela rotina e pelo ritual. O estudo de Alves (1995) mostra que, quatorze anos depois, esta ainda é uma realidade no trabalho na enfermagem pois além da falta de uma cultura de pesquisa nas escolas de enfermagem, a "noção de pesquisa" produz um sentimento de ansiedade em muitos enfermeiros por se sentirem inseguros diante da necessidade de se mostrarem criativos e críticos frente à realidade do trabalho.

Apesar dos avanços, a enfermagem tem investido pouco no processo de produção de conhecimento. Um exemplo disto é a participação muito tímida junto às agências financiadoras desta atividade. O CNPq, por exemplo, oferece alguns espaços para o financiamento da pesquisa mas os projetos da enfermagem encaminhados a esse Conselho são escassos. A participação do norte e nordeste do país é muito discreta. O ano de 1982 foi um marco para o financiamento da pesquisa pois houve um maior estímulo dessa Agência para os cursos de pós graduação e, nos últimos anos, embora os recursos sejam anunciados como escassos, há indícios de possibilidades de aquisição de recursos para a pesquisa. Entretanto, faz-se necessário o envio de projetos a essa e a outras agências 
financiadoras e o seu acompanhamento para ampliação das possibilidades de aquisição de recursos para a pesquisa.

Qual a necessidade de uma base de pesquisa? Walsh (1991).

...acredita que se os enfermeiros querem controlar a enfermagem então deverão ser capazes de justificar as suas ações e decisões e definir os seus próprios padrões e níveis de competências das especialidades apropriadas. Isto exige uma base de conhecimento de enfermagem que, por sua vez, necessita da investigação em enfermagem.

De acordo com Demo (1981), são quatro as linhas básicas de pesquisa: Pesquisa Teórica: aquela que monta e desvenda quadros teóricos de referência; estuda teorias, burila conceitos. Pesquisa Metodológica: dedicada a indagar instrumentos, por caminhos, por modos de se fazer ciência, a produzir técnicas de tratamento da realidade, ou a discutir abordagens teórico-práticas. Pesquisa Empírica: voltada para a face experimental e observável dos fenômenos, manipulando dados e fatos concretos e dedica-se à codificar a face mensurável da realidade. Pesquisa Prática: voltada para intervir na realidade social. Inclui a pesquisa participante e a pesquisa-ação, entre outras.

Fazer qualquer um dos tipos de pesquisa exige um posicionamento do profissional frente às linhas de pensamento existentes as quais definem o que é ciência, quais são seus componentes, critérios de avaliação do que é científico e como se processa a pesquisa. Entre os "pensamentos" mais conhecidos Demo (1981) destaca o empirismo, o positivismo, o funcionalismo sistêmico, a fenomenologia e a dialética. Entretanto, só recentemente esta discussão vem penetrando na enfermagem e um dos espaços para isto tem sido os SENPE. Os anais correspondentes demonstram que a maioria da produção científica na enfermagem baseia-se em uma abordagem positivista embora já exista um número crescente de estudos nas linhas da dialética e da fenomenologia. (Elsen; Bub; Athof, 1991).

Acreditamos que a pesquisa ainda, por mais algum tempo, continuará ocorrendo no "locus" acadêmico e como resultado da produção da pós graduação. Entretanto, mesmo sendo a pesquisa em enfermagem intimamente ligada à pós graduação, observa-se, nos últimos anos, no meio acadêmico, a convergência de pesquisadores em núcleos temáticos ou grupos de pesquisa. Isto vem ocorrendo principalmente após a criação do nível de doutorado. Os enfermeiros doutores vêm se aglutinando em áreas temáticas ou linhas de pesquisa e se integrando a mestres, especialistas, alunos de pós-graduação e de graduação além de bolsistas de pesquisa, para produzirem e divulgarem conhecimento.

Esse contexto mostra que discutir a problemática da pesquisa na realidade brasileira e, em especial, em uma área como a enfermagem que é tida como aplicadora de conhecimentos gerados em outras áreas, é uma tarefa bastante árdua para aqueles que tomam o conhecimento como uma forma de viver essa realidade, ou melhor, de estar no mundo, e que acreditam que as agentes da enfermagem não apenas usam (aplicam) conhecimento mas, também, fazem (produzem) e posicionam-se diante do conhecimento, como indica Alves (1995). 


\section{A PREOCUPAÇÃO DO DEN COM A PESQUISA}

A preocupação do DEN/UFS com a problemática da pesquisa docente e discente vem se ampliando nos últimos anos. Na perspectiva de superar algumas das dificuldades quanto ao ato de pesquisar, esse Departamento vem capacitando seu corpo docente a nível de pós graduação (doutorado, mestrado e especialização) e criando espaços para a difusão dos conhecimentos produzidos. A resposta a esse investimento tem vindo ao longo dos anos, através de uma produção científica composta de livro, pesquisas, artigos diversos e trabalhos apresentados em eventos científicos.

Posturas como essas fazem parte de modificações ocorridas no cenário nacional, em especial nas universidades públicas, que têm "forçado" a comunidade acadêmica a investir mais ousadamente nas atividades de ensino, pesquisa e extensão, de forma integrada. Așsim, apesar de o contexto onde hoje se realiza a pesquisa ser bastante desfavorável, o DEN vem fazendo alguns investimentos no sentido de participar do processo de produção do conhecimento. Desses investimentos, destacamos:

- participação efetiva no processo de tentativa de criação do Doutorado em Enfermagem Inter Universidades do Nordeste;

- criação do Núcleo de Pós Graduação em Enfermagem da UFS e contratação de Professoras Doutoras para se integrar a ele, além do oferecimento do Curso de Especialização em Gerência de Unidade Hospitalar;

- criação do Núcleo de Pós - Graduação em Enfermagem da UFS e contratação de Professoras Doutoras para se integrar a ele, além do oferecimento do Curso de Especialização em Gerência de Unidade Hospitalar;

- formação de mestres e doutores;

- integração com outros cursos de enfermagem, a exemplo da Escola de Enfermagem da UFBA, através de uma Profa. Dra. no seu Programa de Pós Graduação em Enfermagem;

- participação em Comissão de Pesquisa vinculada à Pós - Graduação da UFS;

- identificação de grupos e linhas de pesquisa do Departamento e de projetos de pesquisa concluídos e em andamento;

- estímulo à participação discente na pesquisa através da elaboração de projetos de pesquisa na Disciplina "Metodologia da Pesquisa em Enfermagem", de "Trabalho de Conclusão de Curso - TCC", de "Monografia" durante o Curso de Especialização e de participação de alunas(os) no PIBIC/CNPq/UFS, através de projetos de autoria de docentes.

Embora esse conjunto de estratégias tenha sido apontado como adequado pelas docentes entrevistadas, ele tem sido implementado com muitas dificuldades, dentre as quais destacamos: escasso número de docentes para responder às atividades acadêmicas; dificuldades para afastamento de docentes para processos 
de capacitação, além do difícil acesso ao financiamento da pesquisa na enfermagem, área do conhecimento nem sempre prestigiada pelas políticas de financiamento dos diferentes órgãos fomentadores da pesquisa.

\section{GRUPOS, LINHAS E PROJETOS DE PESQUISA: A CONCEPÇÃO DE DOCENTES DO DEN}

O que proporciona a organização da pesquisa em um trabalho grupal? A pesquisa grupal suscita o aprofundamento de determinadas áreas do conhecimento ou linhas de pesquisa, pois pode-se contar com diversos pesquisadores estudando recortes vários de um mesmo objeto de pesquisa. Permite, ainda, agregar pesquisadores em diversos níveis de conhecimento, como os iniciantes e aqueles que já dominam metodologias específicas e também agregar profissionais de outras áreas. Enfim, proporciona o fortalecimento das linhas de pesquisa e dos grupos que as comportam. As instituições de fomento à pesquisa têm estimulado esse tipo de trabalho: o CNPq tem a modalidade de Projetos Integrados de Pesquisa, a FAPESP tem Projetos Temáticos de Equipe, etc.

Para a organização de grupos de pesquisadores é necessário a titulação de doutores e esse processo tem sido lento na enfermagem. Temos cuidado da titulação dos mestres e descuidado da dos doutores. Um outro elemento importante a ser discutido e que faz parte da organização da pesquisa é a fundamentação teórica e metodológica, conforme apontado por Demo (1981), além da utilização dos resultados de pesquisa na prática e da divulgação desses resultados.

O que significa grupo, linha e projeto de pesquisa? Em relação a grupo de pesquisa, a Escola de Enfermagem da UFBA, segundo Alves; Ferreira (1994), o entende como sendo

...un conjunto de pesquisadores de diferentes níveis, coordenado, preferencialmente, por pesquisador doutor, que se reunem por identidade temática, respeitando, contudo, as diferenças teórico-filosóficas que caracterizam as diversas visoes de mundo desses pesquisadores. O grupo de pesquisa se materializa, num primeiro momento, através de linhas de pesquisa.

No DEN/LFS, a discussão sobre o significado de grupo de pesquisa está sendo posta a partir desta pesquisa. Expressões e palavras extraídas dos depoimentos registrados nos formulários de coleta dos dados, sinalizam para enunciados que definem, em princípio, esta categoria como ...grupo de pesquisadores que se reúnem por identidade temática, tendo como base perspectivas teórico-filosóficas coincidentes ou não.

Em relação a linhas de pesquisa, essas

...são entendidas por Goldderg(1980) ...conio un fio condutor e aglutinador de esforços investigativos, com caráter de cumulatividade e concentração, de um organismo de pesquisa. Elas devem ser concebidas: a)como projeto básico de investigação daquele organismo; b)como o reflexo de uma identidade científica demonstrada pela integração de idéias, interesses, valores e competências do grupo de pesquisadores a 
elas vinculados; c) compromissadas com duas lealdades fundamentais, os problemas brasileiros e os padrões internacionais de produção cientifica; e t)articuladas a projetos congêneres ou correlatos, a nível de comminidade cientifica seja, nacional, seja internacional (Neves; Gonçalves ( 1994, p.224).

Estas mesmas autoras afirmam que, de acordo com o II Seminário Nacional sobre Ensino de Pós - Graduação e Pesquisa em Enfermagem, ... linha de pesquisa em enfermagem compreende 'uma proposta de investigação de saúde da população, realizada de modo progressivo e em condições para imediato engajamento de novos pesquisadores'. Considerando, mais uma vez, a perspectiva da Escola de Enfermagem da UFBA, através do que apresentam Alves; Ferreira(1994), linha de pesquisa ...consiste numa determinada temática que, embora tratada sob diferentes visões de mundo, guarda relação intrínseca com o grupo de pesquisa ao qual se encontra vinculada. Da linha de pesquisa, deriva(m) projeto(s). Para docentes do DEN/UFS, linha de pesquisa refere-se ...a uma temática especial tratada sob perspectivas teórico-filosóficas coincidentes ou não, mas que correspondem a determinado grupo de pesquisa.

Por fim, quanto a última categoria, ou seja, projeto de pesquisa, Alves; Ferreira(1994) assinalam que ele

...procura responder a questões delimitadas, relacionadas com a linha de pesquisa e, consequientemente, com o grupo de pesquisa correspondente. Assim, a vinculação de um projeto a determinada linha de pesquisa passa pela correspondência entre objeto de pesquisa do projeto e interesse temático relativo à linha de pesquisa. Diferentementedo grupo e da linha de pesquisa, o projeto, como parte operacional da pesquisa, está sujeito a prazos previamente definidos.

Para docentes do DEN/UFS, ...o projeto de pesquisa consiste na materialização das idéias previstas em linhas e grupos de pesquisa e procura responder a questões específicas, com prazos determinados.

De acordo com os materiais extraídos das entrevistas com as docentes do DEN/UFS, a expectativa do desenvolvimento da pesquisa nesse Departamento passa pela integração entre grupos, linhas e projetos, internos e externos, ou seja, inter grupos e entre grupos desse Departamento com grupos de pesquisa de outros cursos de enfermagem e/ou de outros cursos dessa Universidade.

\section{PESQUISAS CONCLUÍDAS, PROJETOS EM ANDAMENTO E LINHAS DE PESQUISA DO DEN/UFS}

Segundo informações das docentes entrevistadas foram produzidas, até o momento, 29 pesquisas nas seguintes áreas: saúde da mulher (7); médico-cirúrgica (7); avaliação (7); saúde da criança (3); enfermagem como profissão (2); representação social e enfermagem (2); ética (1) e administração (1). Quanto aos projetos de pesquisa em andamento, foram identificados 11(onze) nas seguintes áreas: história da enfermagem/avaliação (4); saúde da mulher (3 sendo 2 do PIBIC); representação social e enfermagem (2); médico-cirúrgica (1 do PIBIC) e DST/AIDS (1 financiada pelo Banco Mundial). Assim, dos projetos em andamento apenas 4(quatro) têm algum tipo de apoio. Entretanto, essa produção sinaliza quanto às 
possibilidades de constituição de grupos a partir de linhas de pesquisa que se delineiam como saúde da mulher; médico-cirúrgica; representação social e enfermagem; história da enfermagem; avaliação em enfermagem e avaliação curricular.

\section{A PRODUÇÃO CIENTÍFICA DISCENTE COMO PRODUTO DA SOCIALIZAÇÃO DO ATO DE PESQUISAR}

Os cursos de graduação em enfermagem já incluem em seus currículos disciplinas que buscam preparar o aluno para a investigação científica. Entretanto, o currículo oculto cria maior oportunidade ao proporcionar o aprender pesquisar, pesquisando, pois é na convivência com pesquisadores que o aluno tem oportunidade de tirar dúvidas, de analisar textos, de refazer textos mal escritos, e é também junto aos profissionais de serviço que o aluno incorpora a postura de leitor crítico. Outra forma de socialização se dá através da participação em eventos, apresentando trabalhos em co-autoria com colegas e professores orientadores.

No DEN/UFS essa socialização tem acontecido de diferentes formas. Uma delas tem sido a Disciplina "Metodologia da Pesquisa em Enfermagem", que vem buscando introduzir a futura enfermeira no processo de produção de conhecimento através da elaboração de um "Projeto de Pesquisa" como exercício de preparação para o ato de pesquisar. A resposta tem sido satisfatória e já foram produzidos inúmeros projetos embora a quase totalidade tenha sido sob a perspectiva positivista.

Considerando a necessidade de aprofundar esse processo, foi instituído, a partir de 1993, o "Trabalho de Conclusão de Curso - TCC" e, até o momento, já foram elaborados 56. Um destaque especial damos à participação de alunas de graduação no Programa Institucional de Bolsas de Iniciação Científica - PIBIC/CNPq/UFS pois, entre 1994 e 1997, 11(onze) alunas participaram de projetos sob a orientação de docentes do Departamento. Dando prosseguimento a essa filosofia, ao ser criado o Curso de Especialização em Gerência de Unidade Hospitalar, ficou definido que a aluna elaborará uma Monografia.

Esses investimentos demonstram a preocupação do DEN com a introdução das alunas no mundo científico e, conseqüentemente, com a expectativa de ampliação e aprofundamento dessa atividade no Curso de Enfermagem (graduação e pós - graduação) e no processo de trabalho das enfermeiras.

\section{DIFICULDADES RELACIONADAS À PESQUISA}

Apesar de todos os esforços, algumas dificuldades são apresentadas pelas docentes como "obstáculos" ao ato de pesquisar. Entre elas, destacamos:

- pouco investimento na formação de pesquisadores a partir, inclusive, da graduação, o que contribui para a falta de fundamentação teórico-prática quanto à metodologia da pesquisa por parte das docentes de enfermagem;

- quase inexistência de projetos de extensão, como campos privilegiados para o desenvolvimento de práticas subsidiadoras de pesquisas, que contem com a participação efetiva de alunos, professores e outros profissionais da enfermagem e da saúde; 
- existência de poucos grupos de pesquisa, além da sua atuação de forma assistemática;

- existência de grupos não coordenados por pesquisador doutor;

- pouco investimento na integração inter grupos de pesquisa, internos e externos ao Departamento;

- indefinição de linhas de pesquisa;

- não articulação de pesquisadores a partir de linha(s) de pesquisa decorrente(s) das necessidades do ensino e da assistência, em geral;

- execução de projetos de forma assistemática, não havendo clareza quanto a sua vinculação à linha ou grupo de pesquisa;

- dificuldade de acesso às agências financiadoras, tendo como conseqüência o não financiamento da maioria dos projetos de pesquisa;

- dificuldade de acesso aos veículos de publicação;

- falta de tempo para pesquisar;

- falta de material bibliográfico;

- acomodação docente.

\section{PRODUÇÃO CIENTÍFICA DO DEN/UFS: POSSIBILIDADES EM PERSPECTIVA}

Na perspectiva das entrevistadas, as dificuldades apresentadas poderão ser superadas através da criação de possibilidades de ampliação da produção do conhecimento, tais como:

- qualificação docente a nível de doutorado e mestrado;

- formação de pesquisadores;

- (re)organização de grupos de pesquisa com definição de linhas e projetos que guardem relação entre si;

- integração inter grupos do Departamento e entre grupos internos e externos ao Departamento;

- manutenção da obrigatoriedade de elaboração de TCC (Curso de Graduação) e Monografia (Curso de Especialização);

- manutenção da exigência de um projeto de pesquisa na Disciplina "Metodologia da Pesquisa em Enfermagem" e de trabalhos acadêmicos que guardem relação com o tema do TCC, para maior otimização da sua produção e para possibilitar a elaboração de trabalhos que possam ser encaminhados para publicação, favorecendo, conseqüentemente, a sua divulgação;

- busca pelo financiamento da pesquisa;

- maior participação docente e discente no PIBIC/CNPq/UFS.

\section{CONSIDERAÇÕES FINAIS}

Como resposta às necessidades do desenvolvimento da enfermagem, o DEN/UFS vem buscando desenvolver o seu processo de produção do conhecimento 
através da capacitação do seu corpo docente, da organização de grupos, linhas e projetos de pesquisa e da criação de espaços para a difusão dos conhecimentos produzidos, além da introdução do aluno (de graduação e de pós - graduação) no "mundo da pesquisa". Neste estudo, portanto, aparece evidente a necessidade de aprofundamento da investigação científica e da aplicação dos seus resultados na prática de enfermagem.

Para avançar nesse processo, as docentes que participaram desta pesquisa, sugeriram:

- a construção, pelos professores, de grupos de pesquisa a partir da integração

- entre docentes mais experientes e menos experientes, além de alunas;

- o agrupamento das docentes por linhas de pesquisa ou por disciplinas afins;

- a integração com pesquisadores e grupos de pesquisa de outros Departamentos ou Cursos de Enfermagem;

- a definição de linhas de pesquisa do Departamento;

- a maior disponibilidade das docentes para a pesquisa;

- a melhoria da infra estrutura do DEN;

- a realização de seminários sobre pesquisa;

- a identificação, o registro e a divulgação da produção científica do Departamento.

\section{REFERÊNCIAS BIBLIOGRÁFICAS}

1. ALVES, Delvair de Brito Alves. Produção/reprodução do conhecimento no trabalho na enfermagem. Salvador, 1995.245p. Tese (Doutorado) - Faculdade de Educação da Universidade Federal da Bahia.

2. ALVES, Delvair de Brito; FERREIRA, Sílvia Lúcia. A pesquisa na EEUFBA. Revista Baiana de Enfermagem, Salvador, v.8, n.1/2, p.169-76, 1994.

3. CLARK, J.M; HOCKEY, L. Research for nursing. [s.I.] 1989.

4. DEMO, Pedro. Metodologia científica em ciências sociais. São Paulo: Atlas, 1981. $199 p$.

5. ELSEN, Ingrid; BUB, Lydia I.R.; ATHOF, Coleta R. A pesquisa como atividade inerente ao processo de trabalho do enfermeiro. In: SEMINÁRIO NACIONALDEPESQUISA EMENFERMAGEM, 6., 29 de julho a 10 de agosto de 1991. Anais... Rio de Janeiro: ABEn, 1991. p.59-66.

6. GONÇALVES, Ricardo Bruno Mendes. Trabalho em saúde e pesquisa: reflexão a propósito das possibilidades e limites da prática de enfermagem. In: SEMINÁRIO NACIONAL DE PESQUISA EM ENFERMAGEM, 4., 2 a 5 de setembro de 1985. Anais... São Paulo: ABEn, 1985. p.27-39. 
7. NEVES, Eloita Pereira; GONÇALVES, Lúcia H.T. As questões do marco teórico nas pesquisas de enfermagem. In: SEMINÁRIO NACIONAL DE PESQUISA EM ENFERMAGEM, 3., 3 a 6 de abril de 1984. Anais... Florianópolis: ABEn, 1994. p.211-29.

8. SOUZA, Alina Maria de Almeidaetal. Estudo de tendências da pesquisa sobre a prática de enfermagem no Brasil-1983/1987. In: SEMINÁRIONACIONAL DE PESQUISA EM ENFERMAGEM, 5., 25 a 29 de julho de 1988. Anais... Belo Horizonte: ABEn, 1988. p.95-7.

9. WALSH, M. Identifying research for use. Surg Nurse, v.4, n.2, p.25-7, 1991.

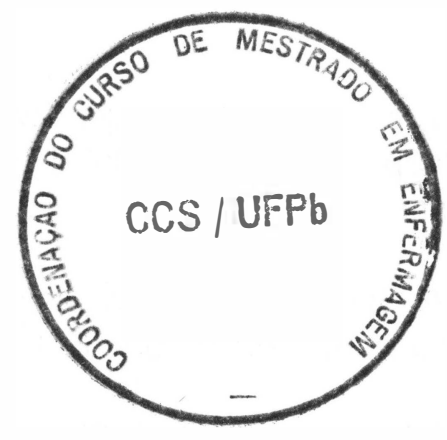

